\title{
Integrating Backstepping Control of Outdoor Quadrotor UAVs
}

\author{
Zsófia Bodó1*, Béla Lantos \\ ${ }^{1}$ Department of Control Engineering and Information Technology, Faculty of Electrical Engineering and Informatics, \\ Budapest University of Technology and Economics, H-1117 Budapest, Magyar Tudósok krt. 2., Hungary \\ *Corresponding author, e-mail: zsbodo@iit.bme.hu
}

Received: 17 October 2018, Accepted: 13 December 2018, Published online: 28 February 2019

\begin{abstract}
In this paper an improved approach is presented for integrating backstepping control of outdoor quadrotor UAVs. The controller uses the approximated nonlinear dynamic model, while for simulation or test purposes the quadrotor can be modeled either with the precise or the simplified model. A hierarchical integrating backstepping control algorithm was constructed that has the capability of handling every effect in the dynamic model and in the meantime successfully ignores the realistic measurement noises. The hierarchical control structure consists of position, attitude and rotor control, extended with path design with continuous acceleration and/or continuous jerk. The state estimation is based on sensor fusion. Control parameters can be easily tuned. Adaptive laws are elaborated for mass and vertical disturbance force estimation. The tracking algorithm is able to follow the prescribed path with small error. The sensory system and the state estimation are prepared for outdoor applications. The embedded control system contains a HIL extension to test the control algorithms before the first flight under real time conditions.
\end{abstract}

\section{Keywords}

kinematic model, dynamic model, quadrotor, integrating backstepping control, embedded realization, sensor fusion, EKF approach, parameter adaptation

\section{Introduction}

The research field of unmanned aerial vehicles (UAVs) is highly prospering these days. Although the field was mainly motivated by the possible military applications, the civilian usage is emerging quickly. Several military and civilian professionals are interested in developing an autonomous mini unmanned outdoor quadrotor helicopter. The benefits of such a system are significant, in the near future the quadrotor may be able to precisely follow a predefined path while performing a measurement series such as performing a surveillance above a predefined territory.

In the control of quadrotor helicopters several control algorithms can be considered, including linear and nonlinear algorithms [1] or soft-computing algorithms [2]. In the domain of nonlinear control algorithms, the most popular technique is the backstepping approach, although several other techniques can be found in this field, including the sliding mode technique [3] and feedback linearization control algorithms [4]. A full state backstepping algorithm is presented in [5].

The paper is organized as the following. Section 2 presents the kinematic and dynamic models of quadrotors. Section 3 describes the standard backstepping control for single variable systems. Section 4 presents the hierarchical backstepping control of UAVs and the cascade controller structure. Section 5 describes the path design and tracking methods. Section 6 presents the adaptive extension for mass and vertical force identification. Section 7 illustrates the efficiency of the developed control approaches using simulation. Section 8 deals with the embedded control realization and the HIL test. Section 9 concludes the paper.

\section{Dynamic model of quadrotors}

\subsection{Kinematic model of aerial vehicles}

It can be assumed that a coordinate system (frame) $K_{E}$ was fixed to the Earth, therefore it might be considered as an inertial frame of reference. In our paper the frame considered to be fixed might be the NED frame.

Another coordinate system fixed to the center of gravity of the quadrotor is $K_{H}$, it can be described by its position $\xi=(x, y, z)^{T}$ and orientation (RPY angles) $\eta=(\Phi, \Theta, \Psi)^{T}$ relative to $K_{E}$. Translational and angular velocities $v$ and $\omega$ of the helicopter are given in $K_{H}$.

The orientation may be described by the (orthonormal) matrix $R_{t}$ as follows: 


$$
R_{t}=\left[\begin{array}{ccc}
C_{\Theta} C_{\Psi} & S_{\Phi} S_{\Theta} C_{\Psi}-C_{\Phi} S_{\Psi} & C_{\Phi} S_{\Theta} C_{\Psi}+S_{\Phi} S_{\Psi} \\
C_{\Theta} S_{\Psi} & S_{\Phi} S_{\Theta} S_{\Psi}+C_{\Phi} C_{\Psi} & C_{\Phi} S_{\Theta} S_{\Psi}-S_{\Phi} C_{\Psi} \\
-S_{\Theta} & S_{\Phi} C_{\Theta} & C_{\Phi} C_{\Theta}
\end{array}\right] .
$$

The relationship between $\dot{\xi}$ and $\dot{\eta}$ and translational and angular velocities $v$ and $\omega$ of the helicopter can be described as

$\dot{\xi}=R_{t} v, \quad \omega=R_{r} \dot{\eta}$

$R_{r}=\left[\begin{array}{ccc}1 & 0 & -S_{\Theta} \\ 0 & C_{\Phi} & S_{\Phi} C_{\Theta} \\ 0 & -S_{\Phi} & C_{\Phi} C_{\Theta}\end{array}\right], R_{r}^{-1}=\left[\begin{array}{ccc}1 & S_{\Phi} T_{\Theta} & C_{\Phi} T_{\Theta} \\ 0 & C_{\Phi} & -S_{\Phi} \\ 0 & S_{\Phi} / C_{\Theta} & C_{\Phi} / C_{\Theta}\end{array}\right]$,

and the time derivative of $\omega$ can be written as

$\varepsilon=\dot{\omega}=R_{r} \ddot{\eta}+\dot{R}_{r} \dot{\eta}$.

Notice that the kinematic model is similar for both fixed wing and quadrotor UAVs.

\subsection{Dynamic model}

The concept of the quadrotor helicopter is shown in Fig. 1. Four brushless DC motors act as the four actuators of the quadrotor helicopter. They exert lift forces $f_{i}$ proportional to the square of the angular velocities $\Omega_{i}$ of the actuators. The rotational directions of the propellers are shown in the sketch.

When applying Newton's laws, the translational and rotational motions of the helicopter in $K_{H}$ may be represented by

$\sum F_{\text {ext }}=m \dot{v}+\omega \times(m v)$

$\sum T_{\text {ext }}=I_{c} \dot{\omega}+\omega \times\left(I_{c} \omega\right)$,

where $I_{c}$ describes the quadcopter's inertia matrix. Let us assume that it can be described by a diagonal matrix $I_{c}=\operatorname{diag}\left(I_{x}, I_{y}, I_{z}\right) . \sum F_{\text {ext }}$ and $\sum T_{\text {ext }}$ describe the forces and torques respectively applied to the quadrotor helicopter expressed in $K_{H}$. These forces and torques are partly caused by the rotation of the rotors ( $F$ and $T$ ), the aerodynamic friction $\left(F_{a}\right.$ and $\left.T_{a}\right)$, the gravitational effect $\left(F_{g}\right)$ in the translational motion and the gyroscopic effect $\left(T_{g}\right)$ in the rotational motion:

$\sum F_{\text {ext }}=F+F_{a}+F_{g}$,

$\sum T_{\text {ext }}=T+T_{a}+T_{g}$

Each of the helicopter's four actuators exert a lift force which is proportional to the square of the angular velocities $\Omega_{i}$ of the actuators $\left(f_{i}=b \Omega_{i}^{2}\right)$. The BLDC motors' reference signals can be programmed in $\Omega_{i}$. The resulting torque and lift force might be described as:

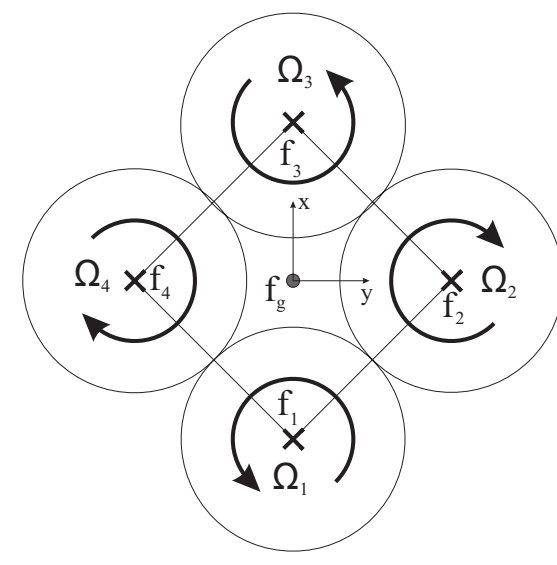

Fig. 1 Concept of the quadrotor helicopter

$$
\begin{gathered}
T=\left(\begin{array}{c}
l b\left(\Omega_{4}^{2}-\Omega_{2}^{2}\right) \\
l b\left(\Omega_{3}^{2}-\Omega_{1}^{2}\right) \\
d\left(\Omega_{2}^{2}+\Omega_{4}^{2}-\Omega_{1}^{2}-\Omega_{3}^{2}\right)
\end{array}\right), \\
f=f_{1}+f_{2}+f_{3}+f_{4}=b \sum_{i=1}^{4} \Omega_{i}^{2},
\end{gathered}
$$

where $l, b, d$ are helicopter and rotor constants. The force $F$ can then be rewritten as $F=(0,0, f)^{T}$.

The gravitational force points to the negative $z$-axis, hence it yields $F_{g}=-m R_{t}^{T}(0,0, g)^{T}=-m R_{t}^{T} G$. The gyroscopic effect can be represented as

$T_{g}=-(\omega \times k) I_{r}\left(\Omega_{2}+\Omega_{4}-\Omega_{1}-\Omega_{3}\right)=-\omega \times\left(k I_{r} \Omega_{r}\right)$.

The rotor inertia value is $I_{r}$ and the third unit vector is $k$.

The aerodynamic friction at low speeds can well be approximated by the linear formulas $F_{a}=-K_{t} v$ and $T_{a}=-K_{r} \omega$.

Using the equations above the motion equations of the quadrotor can be derived:

$$
\begin{aligned}
F= & m R_{t}^{T} \ddot{\xi}-K_{t} R_{t}^{T} \dot{\xi}-m R_{t}^{T} G, \\
T= & I_{c} R_{r} \ddot{\eta}+I_{c}\left(\frac{\partial R_{r}}{\partial \Phi} \dot{\Phi}+\frac{\partial R_{r}}{\partial \Theta} \dot{\Theta}\right) \dot{\eta}+ \\
& +K_{r} R_{r} \dot{\eta}+\left(R_{r} \dot{\eta}\right) \times\left(I_{c} R_{r} \dot{\eta}+k I_{r} \Omega_{r}\right) .
\end{aligned}
$$

\subsection{Simplified dynamic model}

A simplified dynamic model of the quadrotor can be described by disregarding certain effects and thus applying corresponding approximations. For slow helicopter motion all the aerodynamic effects can be neglected. In practice this means that $K_{t}$ and $K_{r}$ might be approximated as zero matrices. Slow motion in lateral directions means little roll and pitch angle changes, therefore $R_{r}$ can be approximated by a 3-by-3 unit matrix. Such simplification cannot be applied to $R_{t}$. 
Consequently, the dynamic equations in Eq. (9) become $F \approx m R_{t}^{T} \ddot{\xi}-m R_{t}^{T} G$,

$T \approx I_{c} \ddot{\eta}+\dot{\eta} \times\left(I_{c} \dot{\eta}+I_{r} \Omega_{r}\right)$.

The six equations are the ones that can be found in $[3,6]$. Because of their importance in later use in the paper they will be repeated here.

$$
\begin{aligned}
m \ddot{x} & \approx\left(C_{\Phi} S_{\Theta} C_{\Psi}+S_{\Phi} S_{\Psi}\right) f, \\
m \ddot{y} & \approx\left(C_{\Phi} S_{\Theta} S_{\Psi}-S_{\Phi} C_{\Psi}\right) f, \\
m \ddot{z} & \approx C_{\Phi} C_{\Theta} f-m g, \\
I_{x} \ddot{\Phi} & \approx \dot{\Theta} \dot{\Psi}\left(I_{y}-I_{z}\right)-I_{r} \dot{\Theta} \Omega_{r}+T_{1}, \\
I_{y} \ddot{\Theta} & \approx \dot{\Psi} \dot{\Phi}\left(I_{z}-I_{x}\right)-I_{r} \dot{\Phi} \Omega_{r}+T_{2}, \\
I_{z} \ddot{\Psi} & \approx \dot{\Phi} \dot{\Theta}\left(I_{x}-I_{y}\right)+T_{3} .
\end{aligned}
$$

\subsection{Rotor dynamics}

Each of the four brushless DC motor's dynamics can be represented as follows:

$$
\begin{gathered}
L \frac{d i_{k}}{d t}=u_{m, k}-R i_{k}-k_{e} \Omega_{k}, \\
I_{r} \dot{\Omega}_{k}=k_{m} i_{k}-k_{r} \Omega_{k}^{2}-k_{s},
\end{gathered}
$$

where $k_{e}, k_{m}$ and $k_{s}$ describes the back emf constant, the motor torque constant and the friction constant, respectively. For negligible motors' inductance Eq. (12) can be transformed to

$$
\begin{aligned}
& \dot{\Omega}_{k}=-k_{\Omega, 0}-k_{\Omega, 1} \Omega_{k}-k_{\Omega, 2} \Omega_{k}^{2}+k_{u} u_{m, k}, \\
& k_{\Omega, 0}=\frac{k_{s}}{I_{r}}, k_{\Omega, 1}=\frac{k_{m} k_{e}}{I_{r} R}, k_{\Omega, 2}=\frac{k_{r}}{I_{r}}, k_{u}=\frac{k_{m}}{I_{r} R} .
\end{aligned}
$$

\section{Standard integral backstepping}

In order to compensate disturbance effects in steady state the usual way is adding the error integrals to the control components. Hence we present first a standard integral backstepping control (IBC) approach which will be used many times in the sequel. By our knowledge the first publication in this field appeared in [7] for motor control and later it became popular also in quadrotor helicopter control.

Let us consider the simple single variable system ( $a$ and $b$ may be nonlinear)

$$
\begin{aligned}
& \dot{x}_{1}=x_{2} \\
& \dot{x}_{2}=a+b u
\end{aligned}
$$

and define the errors and their derivatives as follows:

$$
\begin{aligned}
& e_{1}=x_{1 d}-x_{1} \Rightarrow \dot{e}_{1}=\dot{x}_{1 d}-\dot{x}_{1} \\
& e_{2}=x_{2 d}-x_{2} \Rightarrow \dot{e}_{2}=\dot{x}_{2 d}-\dot{x}_{2}
\end{aligned}
$$

The virtual control $x_{2 d}$ will be chosen as

$$
\begin{aligned}
& x_{2 d}:=c_{1} e_{1}+\lambda \underbrace{\int_{0}^{t} e_{1}(\tau) d \tau}_{p_{1}}+\dot{x}_{1 d} \Rightarrow \\
& \dot{x}_{1 d}=x_{2 d}-c_{1} e_{1}-\lambda p_{1}
\end{aligned}
$$

Then the error derivatives satisfy the following relations:

$$
\begin{aligned}
\dot{e}_{1} & =-c_{1} e_{1}-\lambda p_{1}+e_{2} \\
\dot{e}_{2} & =\dot{x}_{2 d}-\dot{x}_{2}=c_{1} \dot{e}_{1}+\lambda e_{1}+\ddot{x}_{1 d}-(a+b u) \\
& =c_{1}\left(-c_{1} e_{1}-\lambda p_{1}+e_{2}\right)+\lambda e_{1}+\ddot{x}_{1 d}-(a+b u) \\
& =:-c_{2} e_{2}-e_{1} .
\end{aligned}
$$

The form $\dot{e}_{2}=-c_{2} e_{2}-e_{1}$ was prescribed from stability reason. It will be assumed that $c_{1}, \lambda, c_{2}>0$. Using the two equivalent forms of $\dot{e}_{2}$ it follows

$$
\begin{aligned}
& 0=\left(1-c_{1}^{2}+\lambda\right) e_{1}+\left(c_{1}+c_{2}\right) e_{2}-c_{1} \lambda p_{1}+\ddot{x}_{1 d}-(a+b u) \\
& u=\frac{1}{b}\left[\left(1-c_{1}^{2}+\lambda\right) e_{1}+\left(c_{1}+c_{2}\right) e_{2}-c_{1} \lambda p_{1}+\ddot{x}_{1 d}-a\right] .
\end{aligned}
$$

Let us consider now the following Lyapunov function $V$ and its derivative:

$$
\begin{aligned}
V & =\frac{1}{2} \lambda p_{1}^{2}+\frac{1}{2} e_{1}^{2}+\frac{1}{2} e_{2}^{2} \Rightarrow \frac{d V}{d t}=\lambda p_{1} \dot{p}_{1}+e_{1} \dot{e}_{1}+e_{2} \dot{e}_{2} \\
\frac{d V}{d t} & =\lambda p_{1} e_{1}+e_{1}\left(-c_{1} e_{1}-\lambda p_{1}+e_{2}\right)+e_{2}\left(-c_{2} e_{2}-e_{1}\right) .
\end{aligned}
$$

Leaving the canceling terms it remains

$\frac{d V}{d t}=-c_{1} e_{1}^{2}-c_{2} e_{2}^{2} \leq 0$.

Hence, by using LaSalle's stability theorem, the closed loop system with integrator backstepping control is globally asymptotically stable (GAS) if $c_{1}, \lambda, c_{2}>0$.

Algorithm for controller parameter design: Within stability an important question is how to choose the controller's parameters for prescribed speed (closed loop eigenvalues) of the control transients. By Eq. (18) and the use of the control law Eq. (19) the closed loop is

$$
\left[\begin{array}{c}
\dot{e}_{1} \\
\dot{e}_{2} \\
\dot{p}_{1}
\end{array}\right]=\left[\begin{array}{ccc}
-c_{1} & 1 & -\lambda \\
-1 & -c_{2} & 0 \\
1 & 0 & 0
\end{array}\right]\left[\begin{array}{l}
e_{1} \\
e_{2} \\
p_{1}
\end{array}\right],
$$

hence the characteristic equation is

$s^{3}+\left(c_{1}+c_{2}\right) s^{2}+\left(1+c_{1} c_{2}+\lambda\right) s+\lambda c_{2}=0$.

If the closed loop eigenvalues are prescribed by $s_{1}=-a_{1}, s_{2}=-a_{2}, s_{3}=-a_{3}$ and $a_{1} \geq a_{2} \geq a_{3}$ then the characteristic polynomial is

$s^{3}+\left(a_{1}+a_{2}+a_{3}\right) s^{2}+\left(a_{1} a_{2}+a_{1} a_{3}+a_{2} a_{3}\right) s+a_{1} a_{2} a_{3}$ 
and by comparison of the coefficients yields

$c_{2}^{3}-\left(a_{1}+a_{2}+a_{3}\right) c_{2}^{2}+\left(a_{1} a_{2}+a_{1} a_{3}+a_{2} a_{3}-1\right) c_{2}-a_{1} a_{2} a_{3}=0$,

$\lambda=a_{1} a_{2} a_{3} / c_{2}, \quad c_{1}=a_{1}+a_{2}+a_{3}-c_{2}$.

Because of the ordering of the $a_{i}$ values the largest $c_{2}>0$ solution of the cubic polynomial has to be chosen.

Example: If $a_{1}=a_{2}=10$ and $a_{3}=1$ (which is a typical choice in SI units for $T_{s}=0.01 \mathrm{~s}$ sampling time and integral control) then the characteristic polynomial is $s^{3}+21 s^{2}+119 s+100$ and $c_{2}=11.0486, \quad c_{1}=9.9514$ and $\lambda=9.0509$ with the prescribed eigenvalues $\operatorname{eig}(A)=(-10,-10,-1)^{T}$.

\section{Hierarchical backstepping control}

A full state backstepping algorithm is represented in [5] without error integral action. Here the control law is obtained step-by-step through the stabilization of three virtual subsystems and high order derivatives of the path are needed which can cause numerical problems. The helicopter's dynamic model that was shown in Section 2 is comparable to that in the cited paper. In $[3,6,8]$, a backstepping method is applied to the simplified dynamic model of the quadrotor. These serve as the base of the method that is represented in this section.

The next subsections focus on the construction of such an algorithm that is capable of explicitly handling all the effects appearing in Eq. (9), while being ignorant to realistic measurement noises and tolerating disturbances.

\subsection{Cascade controller structure}

Since the helicopter is underactuated, the concept is that the helicopter is required to track a path defined by its $\left(x_{d}, y_{d}, z_{d}, \Psi_{d}\right)$ coordinates. The helicopter's roll and pitch angles are stabilized to 0 internally. The control algorithm can be divided into three main parts. At first, the translational part of the vehicle dynamics is controlled, which then produces the two missing reference signals to the attitude control system. The third part is responsible for generating the input signals of the BLDC motors. The cascade structure of the controller is shown in Fig. 2, where indexes $d$ and $m$ denote desired and measured values, respectively.

Kalman filters can tolerate the difference of measurement frequencies of the position and orientation (GPS or vision system) and acceleration and velocity (IMU). The sampling time of the motor control is set to $10 \mathrm{~ms}$.

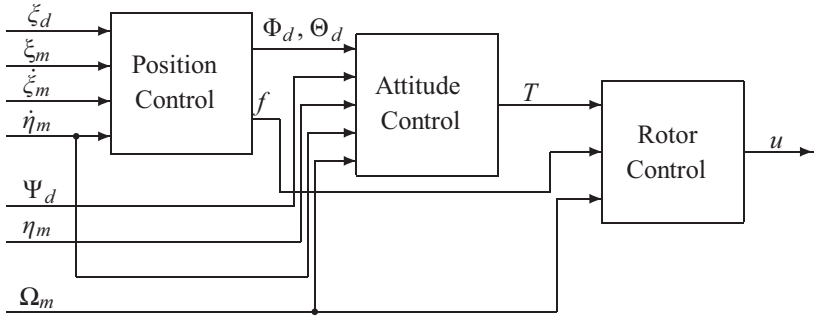

Fig. 2 The cascade structure of the controller

\subsection{Position control}

Using the approximating dynamic model (neglecting aerodynamic friction etc.) the translational motion equations can be brought to the following form:

$$
\left[\begin{array}{c}
\ddot{x} \\
\ddot{y} \\
\ddot{z}
\end{array}\right]=\left[\begin{array}{c}
0 \\
0 \\
-g
\end{array}\right]+\left[\begin{array}{ccc}
\frac{f}{m} S_{\Psi} & \frac{f}{m} C_{\Psi} & 0 \\
-\frac{f}{m} C_{\Psi} & \frac{f}{m} S_{\Psi} & 0 \\
0 & 0 & \frac{C_{\Phi} C_{\Theta}}{m}
\end{array}\right]\left[\begin{array}{c}
S_{\Phi} \\
C_{\Phi} S_{\Theta} \\
f
\end{array}\right] .
$$

As can be seen the standard IBC can be applied for every component of the position vector. Especially for the $z$-component follows

$$
\begin{aligned}
f=\frac{m}{C_{\Phi} C_{\Theta}} & {\left[\left(1-c_{z 1}^{2}+\lambda_{z}\right) e_{z 1}+\left(c_{z 1}+c_{z 2}\right) e_{z_{2}}-c_{z 1} \lambda_{z} p_{z}+\ddot{z}_{d}+g\right], } \\
e_{z 1} & =z_{d}-z \\
e_{z_{2}} & =c_{z 1} e_{z 1}+\lambda_{z} \int_{0}^{t} e_{z 1}(\tau) d \tau+\dot{z}_{d}-\dot{z} .
\end{aligned}
$$

Because of the special form of $\ddot{x}$ and $\ddot{y}$ yields

$$
\begin{aligned}
& \ddot{x}=\frac{f}{m}\left(C_{\Phi} S_{\Theta} C_{\Psi}+S_{\Phi} S_{\Psi}\right)=: \frac{f}{m} u_{x}, \\
& \ddot{y}=\frac{f}{m}\left(C_{\Phi} S_{\Theta} S_{\Psi}-S_{\Phi} C_{\Psi}\right)=: \frac{f}{m} u_{y},
\end{aligned}
$$

It is evident to manage the errors and virtual controls as usual in the standard form, however to express $a$ from the standard form because $b=0$. Therefore we can keep the content of the brackets until the desired accelerations, for example in the first component taking $\left[\ldots+\ddot{x}_{d}\right]_{x}$ and determine from it $u_{\xi_{x}}:=\frac{m}{f}\left[\ldots+\ddot{x}_{d}\right]_{x}$, and similarly $u_{\xi_{y}}:=\frac{m}{f}\left[\ldots+\ddot{y}_{d}\right]_{y}$. Then the desired orientation for the attitude control can be determined from them:

$$
\begin{gathered}
S_{\Phi_{d}}=S_{\Psi} u_{\xi_{x}}-C_{\Psi} u_{\xi_{y}} \Rightarrow \Phi_{d}, \\
S_{\Theta_{d}}=\frac{C_{\Psi} u_{\xi_{x}}+S_{\Psi} u_{\xi_{y}}}{C_{\Phi_{d}}} \Rightarrow \Theta_{d} .
\end{gathered}
$$


The reason why these signals can be considered as reference signals is that as the helicopter approaches the desired coordinates, they converge to zero.

Notice that the errors, error integrals and virtual controls have to be determined separately both in $x$ and $y$ direction according to the standard IBC form.

\subsection{Attitude control}

The design is similar to the standard form and can be applied component-wise. Only the rotation around the $x$-axis will be considered, the other rotations can be managed similarly.

Let us consider the approximating dynamic model as follows (neglecting aerodynamical friction etc.):

$$
\ddot{\Phi}=a_{1} \dot{\Theta} \dot{\Psi}+a_{2} \Omega_{r} \dot{\Theta}+b_{1} \tau_{x}
$$

where $a_{1}=\left(I_{y}-I_{z}\right) / I_{x}, a_{2}=-I_{r} / I_{x}$ and $b_{1}=1 / I_{x}$. Then the IBC control is the following:

$$
\begin{aligned}
\tau_{x}=\frac{1}{b_{1}}[ & \left(1-c_{\Phi 1}^{2}+\lambda_{\Phi}\right) e_{\Phi 1}+\left(c_{\Phi 1}+c_{\Phi 2}\right) e_{\Phi_{2}}-c_{\Phi 1} \lambda_{\Phi} p_{\Phi} \\
& \left.+\ddot{\Phi}_{d}-a_{1} \dot{\Theta} \dot{\Psi}-a_{2} \dot{\Theta} \Omega_{r}\right], \\
e_{\Phi 1}= & \Phi_{d}-\Phi \\
e_{\Phi_{2}}= & c_{\Phi 1} e_{\Phi 1}+\lambda_{\Phi} \underbrace{\int_{0}^{t} e_{\Phi 1}(\tau) d \tau}_{p_{\Phi}}+\dot{\Phi}_{d}-\dot{\Phi} .
\end{aligned}
$$

Similar relations are valid for $\tau_{y}, e_{\Theta_{1}}, e_{\Theta_{2}}$ and $\tau_{z}, e_{\Psi_{1}}, e_{\Psi_{2}}$, respectively:

$$
\begin{aligned}
& \ddot{\Theta}=a_{3} \dot{\Psi} \dot{\Phi}+a_{4} \Omega_{r} \dot{\Phi}+b_{2} \tau_{y} \\
& \ddot{\Psi}=a_{5} \dot{\Phi} \dot{\Theta}+b_{3} \tau_{z}
\end{aligned}
$$

where $\quad a_{3}=\left(I_{z}-I_{x}\right) / I_{y}, \quad a_{4}=-I_{r} / I_{y}, \quad b_{2}=1 / I_{y}$, $a_{5}=\left(I_{x}-I_{y}\right) / I_{z}, b_{3}=1 / I_{z}$.

\subsection{Rotor control}

The calculation of $u_{m}$ differs a little from the method used for inputs. Let us consider one of the rotors and for simplicity suppress the index, then

$$
\begin{aligned}
& \dot{\Omega}_{m}=: f_{m}+g_{m} u_{m} \\
& e_{\Omega}=\Omega_{d m}-\Omega_{m} \\
& \dot{e}_{\Omega}=\dot{\Omega}_{d m}-\left(f_{m}+g_{m} u_{m}\right)=:-c_{\Omega} e_{\Omega}, c_{\Omega}>0 \\
& 0=\left[c_{\Omega} e_{\Omega}+\dot{\Omega}_{d m}-\left(f_{m}+g_{m} u_{m}\right)\right] \\
& u_{m}=\frac{1}{g_{m}}\left[c_{\Omega} e_{\Omega}+\dot{\Omega}_{d m}-f_{m}\right] .
\end{aligned}
$$

For stability proof consider the Lyapunov function $V$ and its derivative:

$$
V=\frac{1}{2} e_{\Omega}^{2} \Rightarrow \frac{d V}{d t}=e_{\Omega} \dot{e}_{\Omega}=-c_{\Omega} e_{\Omega}^{2}<0
$$

Hence the rotor controls are stable. Since the four motors are considered to be identical, $g_{m}$ can be any of $g_{m, k}$-s and therefore it is a scalar. Since $T$ and $f$ are linear combinations of $\Omega_{k_{d}}^{2}$, hence, using symbolic matrix inversion, $\Omega_{k_{d}}$ are the element-wise square roots of the vector at the left side:

$$
\left(\begin{array}{l}
\Omega_{1_{d}}^{2} \\
\Omega_{2_{d}}^{2} \\
\Omega_{2_{d}}^{2} \\
\Omega_{4_{d}}^{2}
\end{array}\right)=\left[\begin{array}{cccc}
0 & -(2 l b)^{-1} & -(4 d)^{-1} & (4 b)^{-1} \\
-(2 l b)^{-1} & 0 & (4 d)^{-1} & (4 b)^{-1} \\
0 & (2 l b)^{-1} & -(4 d)^{-1} & (4 b)^{-1} \\
(2 l b)^{-1} & 0 & (4 d)^{-1} & (4 b)^{-1}
\end{array}\right]\left(\begin{array}{l}
T \\
f
\end{array}\right)
$$

\subsection{State estimation}

The control algorithms need the state variables that may be unmeasured or noisy, hence they have to be estimated from the available sensor measurements. For outdoor applications the state estimation is based on sensor fusion of GPS, IMU and magnetometer. The approach was presented in our earlier paper [9]. The quaternion and 3xEKF (Extended Kalman Filter) based technique can well-tolerate the large difference between IMU and GPS sampling frequencies and can be applied for any type of outdoor vehicles.

The efficiency of the method was demonstrated for real flight data of a fixed wing propeller driven UAV, however the method can also be applied for outdoor quadrotor UAVs considered in this paper. Beside unit quaternion, the orientation (attitude) is also presented in the form of Euler roll, pitch, yaw $(\Phi, \Theta, \Psi)$ angles. The biases of the sensors are online corrected.

\subsection{Parameter tuning of the controller}

The tuning of parameters of the controllers are very simple because only the positivity should be guaranteed. The numerical values are tightly related to the speed and the order of forces and torques influencing the power consumption. It should also be taken into consideration that by increasing the speed of control saturation may be caused in the actuators. Simulation experiments can help in parameter choice based on Eq. (21). 


\section{Path design and tracking}

The typical motion of the quadrotor helicopter can be fit together from takeoff, hovering, attitude change in fixed position and motion along a straight line in fixed direction. These sections must be connected with continuous acceleration or possibly with smooth acceleration (continuous jerk).

In order to spar power the goal is to design path in Cartesian space with continuous/smooth linear and angular accelerations. It can be assumed that the prescribed information for the path is given in the form of the sequences $\{\xi\}_{1}^{n}$ and $\{\Psi\}_{1}^{n}$. Therefore the path information is a sequence of 4-dimensional vectors with scalar components. Hence, the path design problem can be reduced to the path design of a fictitious robot with the joint vector $q=(x, y, z, \Psi)^{T}$ or its subset, then it can be solved by repeating path design in a single scalar variable with bounded and continuous/smooth second order derivative. For the two cases different algorithms will be presented.

\subsection{Path design with continuous acceleration}

The path can be divided into $B^{\prime} \rightarrow B \rightarrow B^{\prime \prime} \rightarrow C$ sections in the normalized time $t \in[-\tau, T]$ where the scalar $y(t)$ is of fourth order if $t \in[-\tau, \tau)$ and linear if $t \in[\tau, T)$. In order to obtain smooth solution it is required

$$
\begin{aligned}
y(t) & =\frac{a_{0}}{12} t^{4}+\frac{a_{1}}{6} t^{3}+\frac{a_{2}}{2} t^{2}+a_{3} t+a_{4} \\
a_{0} & =-\frac{3}{4} \frac{v_{C B}-v_{B B^{\prime}}}{\tau^{3}}, a_{1}=0, a_{2}=\frac{3}{4} \frac{v_{C B}-v_{B B^{\prime}}}{\tau} \\
a_{3} & =\frac{v_{C B}-v_{B B^{\prime}}}{2}, a_{4}=B+\frac{3}{16}\left(v_{C B}-v_{B B^{\prime}}\right) \tau
\end{aligned}
$$

\section{Path design algorithm in q:}

1. Step 1: Prepare the computation of path coefficients:

$$
\begin{aligned}
y_{B^{\prime}} & =q(T-\tau), \quad q_{B}:=q_{C}, \quad q_{C}:=q_{D} \\
T_{i} & =\frac{q_{C_{i}}-q_{B_{i}}}{q^{\prime}}, \quad \forall i \text {, max } \\
T & =\max \left\{\max \left\{T_{i}\right\}, 2 \tau\right\} \\
v_{B B^{\prime}} & :=\frac{q_{B}-q_{B^{\prime}}}{\tau}, \quad v_{C B}:=\frac{q_{C}-q_{B}}{T} .
\end{aligned}
$$

Computation of the coefficients $a_{0}, \ldots, a_{4}$ (can be vectorized). Set the standardized time to $t:=-\tau$.

2. Step 2: Repeat while $t<T-\tau$ :

$$
\begin{aligned}
q(t) & =\left\{\begin{array}{lll}
P_{4}(t) & \text { if } & t \in[-\tau, \tau) \\
P_{1}(t) & \text { if } & t \in[\tau, T-\tau)
\end{array}\right. \\
t & :=t+\Delta .
\end{aligned}
$$

Here $P_{4}(t)$ and $P_{1}(t)$ denote polynomials of given order, $\Delta$ is the step-size (sampling time etc.). The actual path position $\xi_{d}(t)$ comes immediately from the first three components of $q(t)$. The last component of $q(t)$ is defining the desired yaw angle $\Psi_{d}(t)$ for the orientation. The remaining Euler angles $\Phi_{d}(t)$ and $\Theta_{d}(t)$ are the result of real time computations (see above) and the desired orientation matrix can be determined by substituting them into $R_{t}(t)$.

\subsection{Path design with continuous jerk}

In case of the motion along a straight line in fixed direction, the yaw angle $\Psi_{d}$ must be constant on the traveling portion instead of to be linear as above, while the acceleration has to be smooth, i.e. the jerk is continuous. For this purpose a special path design is suggested where the scalar $y(t)$ is of fifth order satisfying

$$
\begin{aligned}
y(t) & =\frac{a_{0}}{60} t^{5}+\frac{a_{1}}{24} t^{4}+\frac{a_{2}}{6} t^{3}+\frac{a_{3}}{2} t^{2}+a_{4} t+a_{5} \\
\ddot{y}\left(-\tau_{0}\right) & =\ddot{y}\left(\tau_{0}\right)=0, \quad \dot{y}\left(-\tau_{0}\right)=\dot{y}\left(\tau_{0}\right)=0, \\
y\left(-\tau_{0}\right) & =q_{B}, \quad y\left(\tau_{0}\right)=q_{C} \Rightarrow a_{1}=a_{3}=0, \\
a_{0} & =\frac{45}{4} \frac{q_{C}-q_{B}}{\tau_{0}^{5}}, \quad a_{2}=-\frac{15}{4} \frac{q_{C}-q_{B}}{\tau_{0}^{3}}, \\
a_{4} & =\frac{15}{16} \frac{q_{C}-q_{B}}{\tau_{0}}, \quad a_{5}=\frac{1}{2}\left(q_{C}+q_{B}\right) .
\end{aligned}
$$

Due to practical consideration $\tau_{0}=n \cdot \tau$ is allowed where $n$ is an integer number. The main difference to the previous algorithm is that here $y(t)=q_{C}$ is constant on the traveling part.

Since the path evaluations are performed in normalized time, hence a precise technique was elaborated to convert paths obtained for different $\tau$ and $\tau_{0}$ values to absolute time by taking into consideration also the sampling time, such that the desired paths remains continuous/smooth. Notice that small spikes in the acceleration could cause large torque/force signals.

\subsection{The tracking algorithm with filtering and multiple differentiation}

The purpose of the control design is to track a predefined trajectory with the smallest possible tracking error. In practice a navigation point must be approximated with a predefined accuracy considering the positions and orientations.

The conditions for position tracking ensures that the helicopter will remain in the proximity of the navigation points while keeping the motion continuous which is supported by the path design. 
On the other hand, the orientation control algorithm needs the derivatives of $\Phi_{d}$ and $\Theta_{d}$ by the time. For robust filtering and differentiation a fictitious control system (integrator plant $1 / s$, first order serial compensator $F_{1} /\left(s+F_{2}\right)$ and outer unity feedback) was designed. Denote $\alpha$ anyone of the two angles to be differentiated, then

$\dot{\alpha}_{1}=\alpha_{2}$

$\dot{\alpha}_{2}=F_{1}\left(r-\alpha_{1}\right)-F_{2} \alpha_{2}$

$y_{1}=\alpha_{1}$

$y_{2}=\alpha_{2}$

where $r$ is the input signal to be differentiated, $y_{1}$ is the filtered signal and $y_{2}$ is the numerically differentiated input. The obtained term can be cascaded considering $y_{2}$ as the input for the next term. Then the state equation of the composite member is as follows:

$\dot{\alpha}_{1}=\alpha_{2}$

$\dot{\alpha}_{2}=-F_{1} \alpha_{1}-F_{2} \alpha_{2}+F_{1} r$

$\dot{\alpha}_{3}=\alpha_{4}$

$\dot{\alpha}_{4}=F_{1} \alpha_{2}-F_{1} \alpha_{3}-F_{2} \alpha_{4}$

$y_{1}=\alpha_{1}$

$y_{2}=\alpha_{2}$

$y_{3}=\alpha_{4}$

where $y_{1}$ is the filtered input $r f, y_{2}$ is the first derivative $d r$ and $y_{3}$ is the second derivative $d d r$.

\section{Adaptive control}

The standard IBC can be extended in the direction of parameter and disturbance force identification.

\subsection{Modeling the parameter changes}

Denote $\theta=\hat{\theta}+\tilde{\theta}$ the unknown parameter vector then it follows:

$$
\begin{aligned}
& a+b u=\hat{a}+\hat{b} u+\underbrace{\sum_{i} \frac{\partial a}{\partial \theta_{i}}\left|\hat{\theta}_{i} \tilde{\theta}_{i}+\sum_{i} \frac{\partial b}{\partial \theta_{i}}\right| \hat{\theta}_{i} \tilde{\theta}_{i} u}_{\tilde{a}+\tilde{b}_{u}} \\
& u:=\frac{1}{\hat{b}}\left[\left(1-c_{1}^{2}+\lambda_{1}\right) e_{1}+\left(c_{1}+c_{2}\right) e_{2}-c_{1} \lambda_{1} p_{1}+\ddot{x}_{1 d}-\hat{a}\right] .
\end{aligned}
$$

Prescribing as usual from stability point of view $\dot{e}_{2}:=-c_{2} e_{2}-e_{1}$ then

$$
\dot{e}_{2}+c_{2} e_{2}+e_{1}=[\ldots-\hat{a}-\hat{b} u-\tilde{a}-\tilde{b} u] .
$$

Taking into consideration the chosen form of $u$ and canceling the equivalent terms we obtain for the parameter identification the relation: $\dot{e}_{2}=-c_{2} e_{2}-e_{1}-\sum_{i} \frac{\partial a}{\partial \theta_{i}}\left|\hat{\theta}_{i} \tilde{\theta}_{i}+\sum_{i} \frac{\partial b}{\partial \theta_{i}}\right| \hat{\theta}_{i} \tilde{\theta}_{i} u$

From application point of view, a typical reason of parameter change is the change of the mass. Such situation arises if some load will be dropped (parcel, food etc.) in civil application or some missile will be fired in military application.

In both cases the remaining mass of the quadrotor is changing which will have an influence to the control properties. It will be assumed that the change of the center of gravity (COG) can be neglected or can be considered as a vertical disturbance, and the controller is able to reprogram itself if this information is available.

\subsection{Mass and vertical force identification}

Let us consider the identification of the mass $m$ and a disturbance force $D_{z}$ :

$$
\begin{aligned}
& \ddot{z}=\underbrace{-g+\frac{D_{z}}{m}}_{a}+\underbrace{\frac{C_{\Phi} C_{\Theta}}{m}}_{b} u \\
& m=\hat{m}+\tilde{m} \Rightarrow \dot{m}=0=\dot{\hat{m}}+\dot{\tilde{m}} \Rightarrow \dot{\tilde{m}}=-\dot{\hat{m}} \\
& D_{z}=\hat{D}_{z}+\tilde{D}_{z} \Rightarrow \dot{D}_{z}=0=\dot{\hat{D}}_{z}+\dot{\tilde{D}}_{z} \Rightarrow \dot{\tilde{D}}_{z}=-\dot{\hat{D}}_{z} .
\end{aligned}
$$

Using the general results above, it follows:

$$
\begin{aligned}
\frac{\partial a}{\partial m} \tilde{m} & =-\frac{1}{(\hat{m})^{2}} \hat{D}_{z} \hat{m}, \quad \frac{\partial a}{\partial D_{z}} \tilde{D}_{z}=\frac{1}{\hat{m}} \tilde{D}_{z} \\
\frac{\partial b}{\partial m} \tilde{m} u & =-\frac{1}{(\hat{m})^{2}} \tilde{m} C_{\Phi} C_{\Theta} u \\
& =-\frac{C_{\Phi} C_{\Theta}}{(\hat{m})^{2}} \frac{\hat{m}}{C_{\Phi} C_{\Theta}}[\ldots+\ddot{z}_{d}-\underbrace{\left(-g+\frac{\hat{D}_{z}}{\hat{m}}\right)}_{\hat{a}}] \tilde{m} \\
\dot{e}_{z 2} & =-c_{z 2} e_{z 2}-e_{z 1}+\frac{\tilde{m}}{\hat{m}} \frac{\hat{D}_{z}}{\hat{m}}-\frac{\tilde{D}_{z}}{\hat{m}} \\
& +\left[\ldots+\ddot{z}_{d}+g-\frac{\hat{D_{z}}}{\hat{m}}\right] \frac{\tilde{m}}{\hat{m}} \Rightarrow \\
\dot{e}_{z 2} & =-c_{z 2} e_{z 2}-e_{z 1}-\frac{\tilde{D}_{z}}{\hat{m}}+\left[\ldots+\ddot{z}_{d}+g\right]
\end{aligned}
$$

Using Lyapunov theory:

$$
V=\frac{1}{2} \lambda_{z} p_{z}^{2}+\frac{1}{2} e_{z 1}^{2}+\frac{1}{2} e_{z 2}^{2}+\frac{1}{2 \gamma_{z 1}} \frac{1}{\hat{m}} \tilde{m}^{2}+\frac{1}{2 \gamma_{z 2}} \frac{1}{\hat{m}} \tilde{D}_{z}^{2}
$$

$\frac{d V}{d t}=\lambda_{z} p_{z} e_{z 1}+e_{z 1} \dot{e}_{z 1}+e_{z 2} \dot{e}_{z 2}-\frac{\tilde{m}}{\gamma_{z 1} \hat{m}} \dot{\hat{m}}-\frac{\tilde{D}_{z}}{\gamma_{z 2} \hat{m}} \dot{\hat{D}}_{z}$.

Substituting $\dot{e}_{z 1}=-c_{z 1} e_{z 1}-\lambda_{z} p_{z}+e_{z 2}$ from standard theory and $\dot{e}_{z 2}$ from above it follows: 


$$
\begin{aligned}
\frac{d V}{d t} & =-c_{z_{1}} e_{z_{1}}^{2}-c_{z_{2}} e_{z_{2}}^{2} \\
& +\frac{\tilde{m}}{\hat{m}}\left\{e_{z_{2}}\left[\left(1-c_{z_{1}}^{2}+\lambda_{z 1}\right) e_{z 1}+\ldots+\ddot{z}_{d}+g\right]-\frac{1}{\gamma_{z 1}} \dot{\hat{m}}\right\} \\
& +\frac{\tilde{D_{z}}}{\hat{m}}\left\{-e_{z_{2}}-\frac{1}{\gamma_{z 2}} \dot{\hat{D}}_{z}\right\} .
\end{aligned}
$$

Making the braces to zero then the stability condition is satisfied by $\frac{d V}{d t}=-c_{z 1} e_{z 1}^{2}-c_{z 2} e_{z 2}^{2} \leq 0$ and an adaptation law is obtained for mass and disturbance force identification. Adaptation law:

$$
\dot{\hat{m}}=\gamma_{z_{1}} e_{z_{2}}\left[\left(1-c_{z_{1}}^{2}+\lambda_{z_{1}}\right) e_{z_{1}}+\left(c_{z_{1}}+c_{z_{2}}\right) e_{z_{2}}-c_{z_{1}} \lambda_{z_{1}} p_{z}+\ddot{z}_{d}+g\right]
$$

$\dot{\hat{D}}_{z}=-\gamma_{z_{2}} e_{z_{2}}$

\section{Simulation tests}

The mechanical parameters of the helicopter and the BLDC motors with the rotors are based on the planned dimensions, the masses of purchased elements. These values are summarized in Table 1.

For path design with continuous acceleration (in $x, y, z$ directions) $\tau=5$ and $\dot{q}_{i, \max }=0.07$ were chosen, while for path design with continuous jerk (only for $\Psi$ ) $\tau_{0}=5 \tau$ and traveling time $T$ according to the values obtained for $x, y, z$ directions were chosen (all values are in SI units).

\subsection{IBC with known system parameters}

As a final result of the integration of all the components of the integrating backstepping control (IBC) the tracking of a complex trajectory consisting of a general 3D line followed by a pentagon in horizontal plane is presented in Fig. 3 for known helicopter mass.

\subsection{Estimation of the initial helicopter mass}

In some cases the initial mass of the helicopter is unknown, for example because it was changed after the last flight. Hence the parameter estimation can help to obtain reliable values of the mass for the IBC control. The estimation results are shown in Fig. 4 for unknown helicopter mass. The starting value was $1.5 m_{\text {real }}$.

Beside the mass estimation also the position trajectory and the errors $e_{z 1}, e_{z 2}$ and $\int e_{z 1}$ are shown because of their influence of the helicopter behavior. It can be seen that the position in $z$-direction can become zero (and also negative), hence the integration of the $e_{z 1}$ component should be switched out for large errors. The signal IMODE shows whether the integration is running (1) or switched out (0). This concept is applied also for other control situations.

\begin{tabular}{lc}
\multicolumn{2}{c}{ Table 1 The physical parameters of the helicopter and the motors } \\
\hline Parameter & Value \\
\hline$l$ & $0.23 \mathrm{~m}$ \\
$b$ & $1.759 \cdot 10^{-5} \mathrm{~kg} \mathrm{~m}$ \\
$d$ & $1.759 \cdot 10^{-6} \mathrm{kgm}^{2}$ \\
$I_{x}, I_{y}$ & $1.32 \cdot 10^{-2} \mathrm{kgm}^{2}$ \\
$I_{z}$ & $2.33 \cdot 10^{-2} \mathrm{kgm}^{2}$ \\
$I_{r}$ & $5.626 \cdot 10^{-5} \mathrm{kgm}^{2}$ \\
$m$ & $1.4 \mathrm{~kg}^{2}$ \\
$K_{t}$ & $\operatorname{diag}([0.1,0.1,0.15]) \mathrm{Ns} / \mathrm{m}$ \\
$K_{r}$ & $\operatorname{diag}([0.1,0.1,0.15]) \mathrm{Nsm}$ \\
$k_{\Omega, 0}$ & $94.37 \mathrm{~s}^{-2}$ \\
$k_{\Omega, 1}$ & $3.02 \mathrm{~s}^{-1}$ \\
$k_{\Omega, 2}$ & 0.005 \\
$k_{u}$ & $139.44 \mathrm{~V} / \mathrm{s}^{2}$ \\
\hline
\end{tabular}

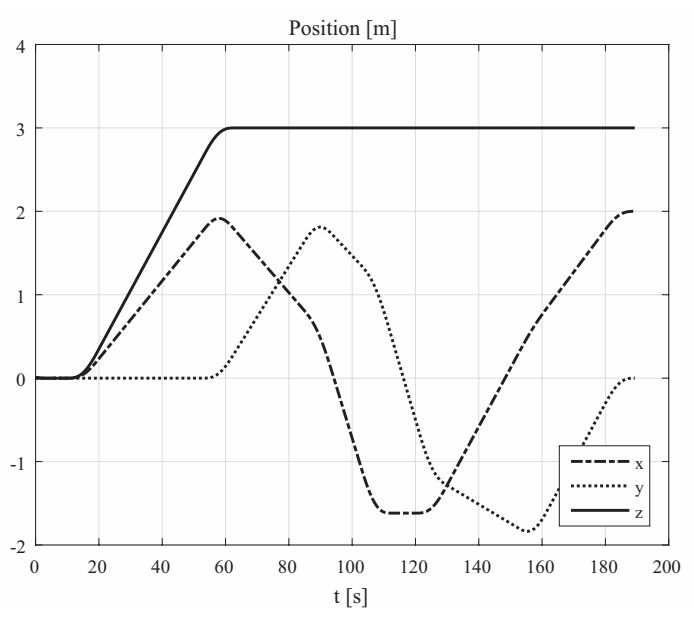

(a) Position

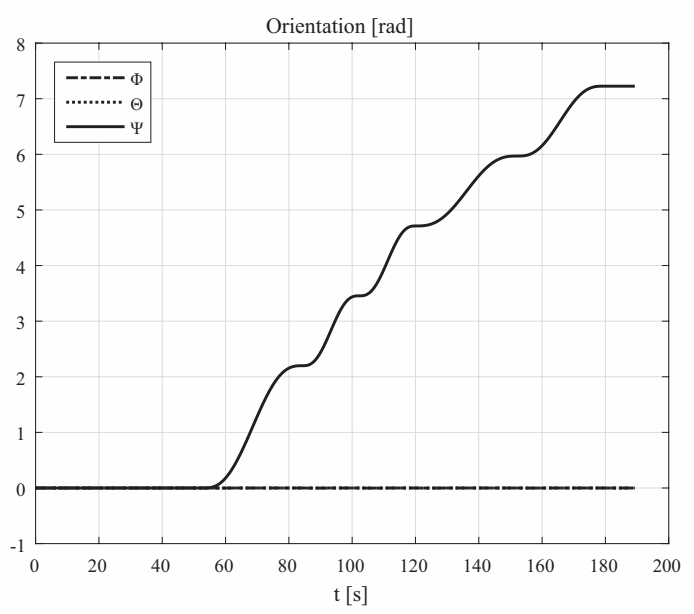

(b) Attitude

Fig. 3 The position and attitude of the helicopter using IBC control for known helicopter mass 

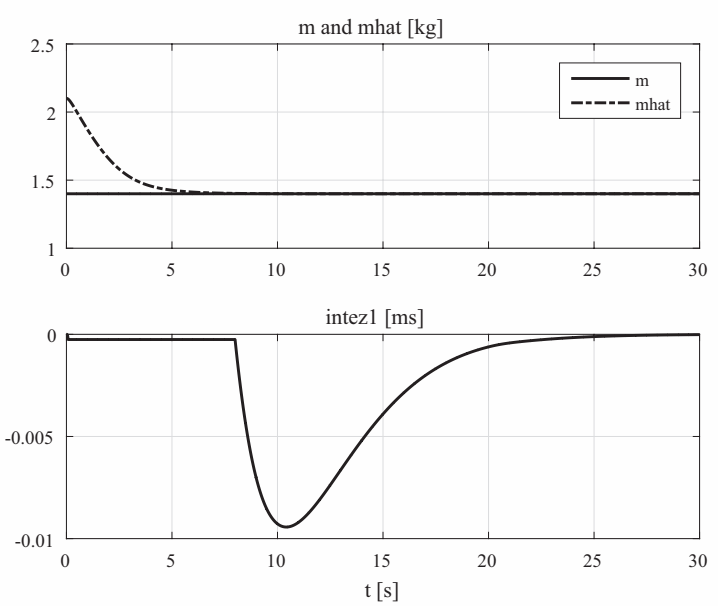

(a) Mass Estimation
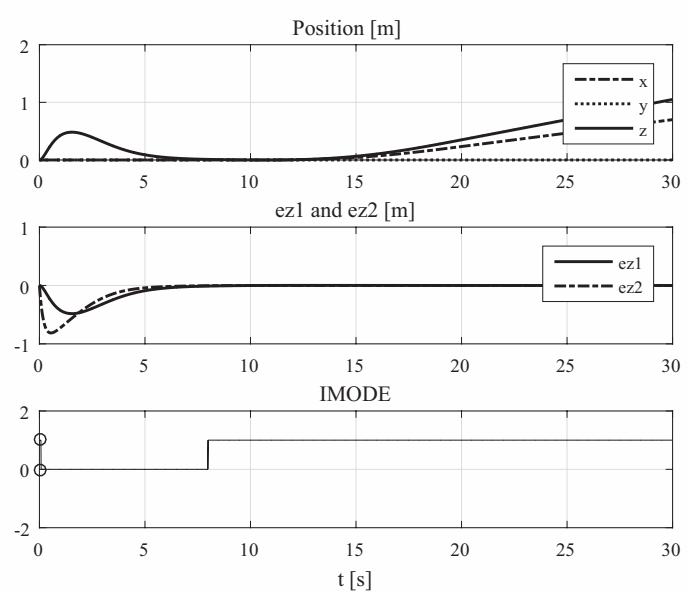

(b) Integral Error

Fig 4 Estimation of the initial helicopter mass and the order of errors

\subsection{Estimation of varying mass and vertical disturbance force}

The estimation results of the varying mass and the vertical disturbance force are shown in Fig. 5 together with the position and the integral errors. The error integration is switched out if $e_{z 1}$ is larger than $0.01 \mathrm{~m}$ which is typical during the initial phase of parameter estimation.

\subsection{Attitude and Rotor Control}

The desired $\Phi_{d}$ and $\Theta_{d}$ is produced by the position control system and has to be differentiated several times by the cascaded fictitious control systems for use in the attitude IBC control.

The rotor controls have also IBC principle but some modifications were necessary because of the (only) first order dynamics. Controller parameter design for IBC rotor control was discussed earlier.

The attitude control transients and the angular velocities and driving torques of the motors are shown in Fig. 6.
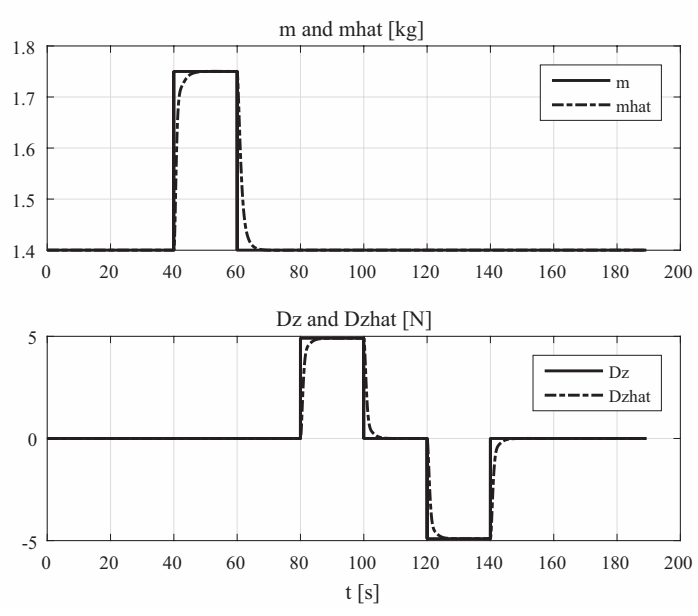

(a) Mass and Force Estimation
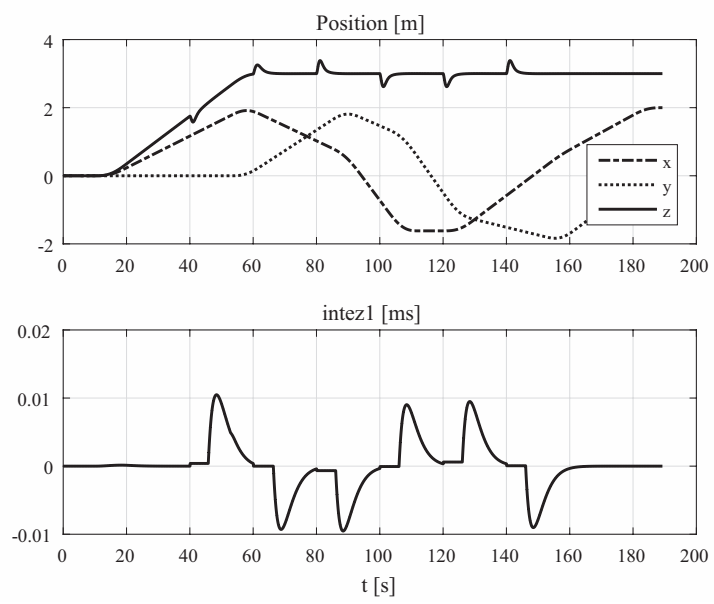

(b) Position and Integral Error

Fig. 5 Estimation of the mass and the vertical disturbance force

\section{Embedded Control Realization}

The hierarchical structure of the controller has already been shown in Fig. 2.

\subsection{Control architecture}

For embedded realization a hardware architecture was developed that is shown in Fig. 7.

The control loop of the helicopter requires accurate position and orientation information. A primary sensor for this is Crossbow MNAV100CA containing GPS and inertial measurement unit (IMU) which provides 3D acceleration and angular velocity and magnetometer measurements together with pressure and temperature information. It contains also 9 servo PWM outputs (not used here). Crossbow's MICRO-VIEW software is also included to assist users with calibration, control, data collection and overall system development.

Part of the architecture is the motor control unit. The rotors are driven by brushless DC (BLDC) motors. The 

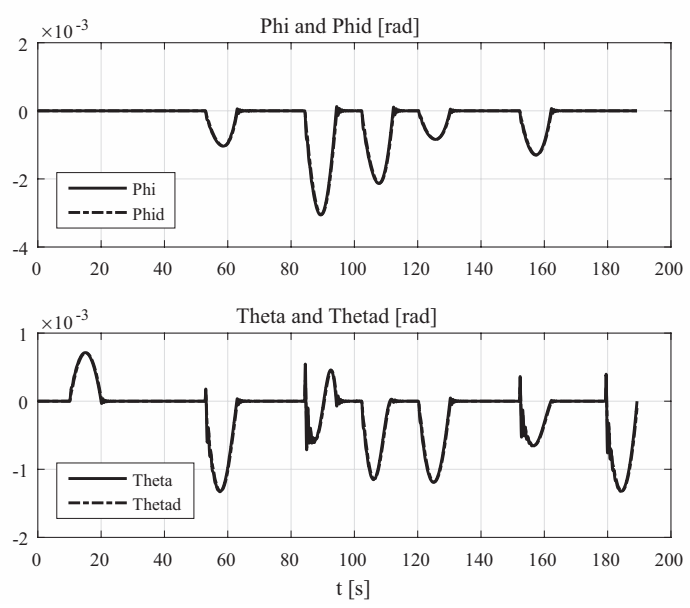

(a) Phi and Theta Control
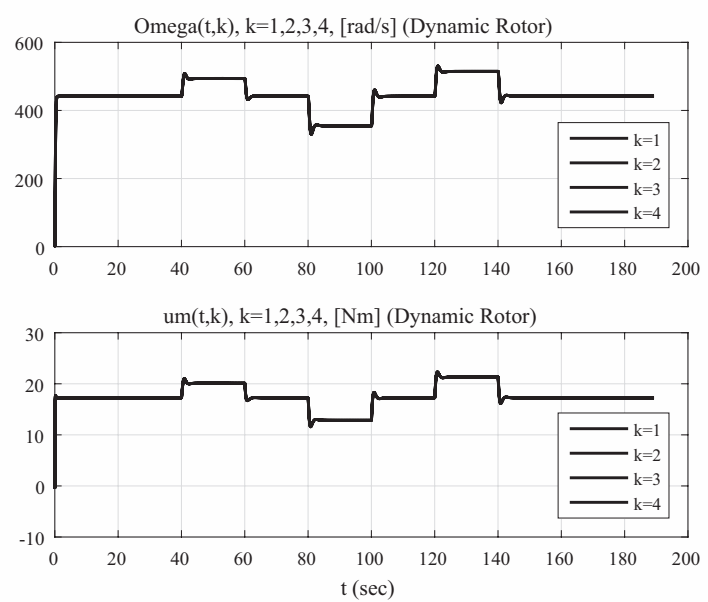

(b) Angular Velocity and Torque Control

Fig. 6 Attitude and Rotor Control

motor controllers and the sensory unit are connected to the CPU via CAN bus.

The on-board computer is a phyCORE-MPC555 equipped with a floating point unit and the controller can be developed at MATLAB/Simulink level. The internal bus is CAN 2.0. The hardware architecture contains an RF channel providing bidirectional communication between the quadrotor and the ground station including also a camera unit. The ground station sends commands and reference path information to the CPU. The helicopter sends status information to the ground.

\subsection{Hardware-in-the-loop test}

Because of the complexity of flying systems, it is inevitable to verify their control systems thoroughly before flight. Before implementing the control algorithm on the embedded target, it was tested using hardware-in-theloop method (HIL). The tests were aided by a dSPACE DS1103 board on which the helicopter model and the

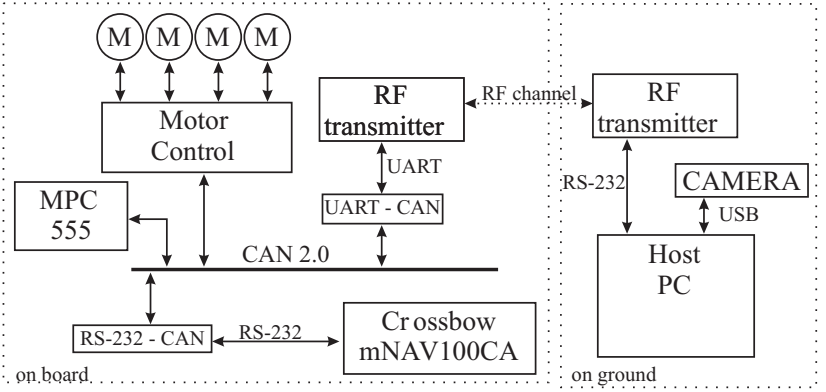

Fig. 7 Embedded controller architecture

sensory system's measurements were emulated while further experiments included the real control architecture and software realizing the control algorithm and the $3 x E K F$ based state estimation.

The scheme of the HIL test can be found in Chapter 6 of [10].

\section{Conclusions}

The paper has been dealt with the problem of modeling and control of outdoor quadrotor helicopters (UAVs). Novelties of the paper are the following:

1. A hierarchical integrating backstepping based nonlinear algorithm was elaborated for stabilizing and path tracking including controller parameter design.

2. This paper's results differ from earlier ones in the tuning of the stabilizing controllers and that they are integrated with a novel quaternion based approach of state estimation usable for any type of vehicles.

3. Adaptation laws are presented for mass and disturbance force estimation.

4. A technique was elaborated for switch in/out of the integrators in the several IBC controllers based on the norm of the error which can increase especially during parameter estimation.

5. An embedded control architecture was suggested which is general enough for many types of vehicles.

Future research will deal with the control of fixed wing UAVs based on the developed control architecture and sensory system.

\section{Acknowledgement}

The work of Zs. Bodó in this paper was supported by the Higher Education Excellence Program of the Ministry of Human Capacities in the frame of Artificial Intelligence research area of Budapest University of Technology and Economics (BME FIKP-MI/FM). 


\section{References}

[1] Bouabdallah, S., Noth, A., Siegwart, R. "PID vs LQ control techniques applied to an indoor micro quadrotor", In: 2004 IEEE/ RSJ International Conference on Intelligent Robots and Systems (IROS) (IEEE Cat. No.04CH37566), Sendai, Japan, 2004, pp. 2451-2456.

https://doi.org/10.1109/IROS.2004.1389776

[2] Pozna, C., Minculete, N., Precup, R.-E., Kóczy, L. T., Ballagi, Á. "Signatures: Definitions, operators and applications to fuzzy modelling", Fuzzy Sets and Systems, 201, pp. 86-104, 2012. https://doi.org/10.1016/j.fss.2011.12.016

[3] Bouabdallah, S., Siegwart, R. "Backstepping and Slidingmode Techniques Applied to an Indoor Micro Quadrotor", In: Proceedings of the 2005 IEEE International Conference on Robotics and Automation, Barcelona, Spain, 2005, pp. 2247-2252. https://doi.org/10.1109/ROBOT.2005.1570447

[4] Das, A., Subbarao, K., Lewis, F. "Dynamic inversion of quadrotor with zero-dynamics stabilization", In: 2008 IEEE International Conference on Control Applications, San Antonio, TX, USA, 2008, pp. 1189-1194.

https://doi.org/10.1109/CCA.2008.4629582

[5] Madani, T., Benallegue, A. "Control of a Quadrotor MiniHelicopter via Full State Backstepping Technique", In: Proceedings of the 45th IEEE Conference on Decision and Control, San Diego, CA, USA, 2006, pp. 1515-1520.

https://doi.org/10.1109/CDC.2006.377548 